\title{
Primary Amenorrhea with Mayer-Rokitansky-Kuster-Hauser Syndrome
}

\author{
Muhammad Rusda ${ }^{1 *}$, Delfi Lutan ${ }^{1}$, Mervina Andarini ${ }^{1}$, Andrina Yunita M. Rambe ${ }^{2}$ \\ ${ }^{1}$ Department of Obstetrics and Gynaecology, Division of Reproductive Endocrinology and Infertility, Medical Faculty, Universitas \\ Sumatera Utara, RSUP Haji Adam Malik, Medan, Indonesia; ${ }^{2}$ Department of Otorhinolaryngology, Medical Faculty, Universitas \\ Sumatera Utara, RSUP Haji Adam Malik, Medan, Indonesia
}

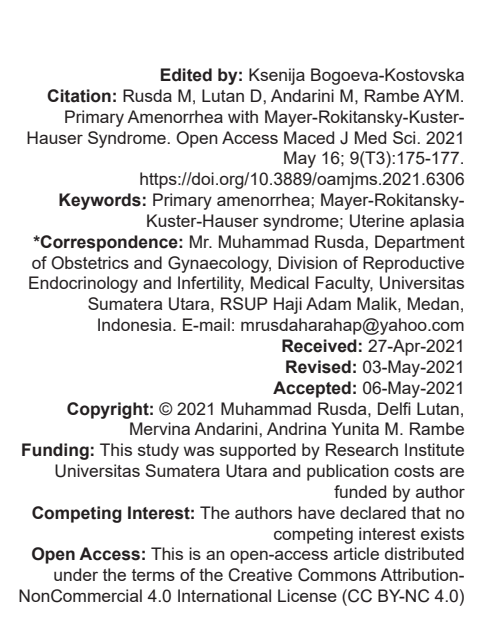

\section{Abstract}

BACKGROUND: Mayer-Rokitansky-Kuster-Hauser (MRKH) syndrome is a rare disorder described as aplasia or hypoplasia of uterus and vagina due to an early arrest in the development of Mullerian ducts. The etiology of MRKH syndrome remains uncertain: Although at the beginning, it was mentioned that this syndrome was the result of sporadic abnormalities. It has recently been assumed the genetic background is the cause of the increasing number of familial cases.

CASE REPORT: A 29-year-old female patient presented with amenorrhea. She also has normal pubic and axillary hair and breast development. Routine investigations were normal. Genitalia inspection shows that pubic hair was well developed, labia majora and vagina were seen. Inspeculo show that vaginal wall was smooth, but portio was not seen. Vaginal examination result: The uterus was unpalpable, both adnexa were normal, parametrium was laxed, and Douglas pouch was not protruded. Rectal examination shows that uterus and both adnexa were difficult to identify. Transvaginal sonography revealed absence of uterus, both ovaries within normal. Transvaginal sonography diagnosed a uterine aplasia. With this, the provisional diagnosis made as primary amenorrhea due to suspected MRKH syndrome, the patient was referred for follicle-stimulating hormone, estradiol, testosterone, and prolactin levels by enzyme-linked immunosorbent assay examination and the result was within normal limit followed by genetic karyotyping and the result was normal $46, X X$.

CONCLUSIONS: We report a 29-year-old woman with primary amenorrhea. From our examination, the patient was diagnosed with MRKH syndrome. Uterus transplant is an alternative for that woman to treat amenorrhea and possibility to get pregnant.

\section{Introduction}

In clinical practice, infertility may encounter female patients which may cause by primary or secondary causes. In the group of primary infertility, although the incidence is relatively small, congenital anomalies are significantly affected. Mayer-RokitanskyKuster-Hauser syndrome (MRKH) is characterized by congenital absence of the uterus and vagina. There is a failure of development of the Mullerian derivative of the vagina and the portions of the Mullerian ducts that form the uterus. The incidence is $1 / 5000$ female births [1]. There are two types of MRKH syndrome: Type 1 shows isolated variation, while type 2 is associated with several organic abnormalities involving upper urinary tract ( $40 \%$ of cases), skeleton (10-12\% cases), hearing system $10-25 \%$ of cases), and rarely involving cardiac system. The etiology of MRKH syndrome remains uncertain: Although at the beginning, it was mentioned that this syndrome was the result of sporadic abnormalities. It has recently been assumed the genetic background is the cause of the increasing number of familial cases [1].
MRKH syndrome is the second most common cause of primary amenorrhea after gonadal dysgenesis. Women with this syndrome are characterized by the presence of $46 \mathrm{XX}$ karyotype, normal female secondary sex characters, normal ovarian functions, and underdeveloped vagina [1]. This is a case report of MRKH syndrome from the uterus in 29 years, married and phenotypically female patient.

\section{Case Report}

A 29-year-old female patient presented with amenorrhea. The patient had no history of abdominal lump or leukorrhea. She also has normal pubic and axillary hair and breast development. Micturition and defecation show no abnormality. Routine investigations were normal. Genitalia inspection shows that pubic hair was well developed, labia majora and vagina were seen. Inspeculo show that vaginal wall was smooth, but portio was not seen. Vaginal examination result: The uterus was unpalpable, both adnexa 
were normal, parametrium was laxed, and Douglas pouch was not protruded. Rectal examination shows that uterus and both adnexa were difficult to identified, anal sphincter was tight, rectal mucosa was smooth, ampulla recti not fulfilled. Transvaginal sonography revealed absence of uterus, both adnexa were normal, right ovary within normal limit with seven follicles, left ovary also within normal limit with five follicles. Free fluid collection was not found. Transvaginal sonography diagnosed a uterine aplasia (Figure 1). With this, the provisional diagnosis made as primary amenorrhea due to suspected MRKH syndrome, the patient was referred for follicle-stimulating hormone (FSH), estradiol, testosterone, and prolactin levels by enzyme-linked immunosorbent assay examination and the result was within normal limit followed by genetic karyotyping and the result was normal 46, XX.

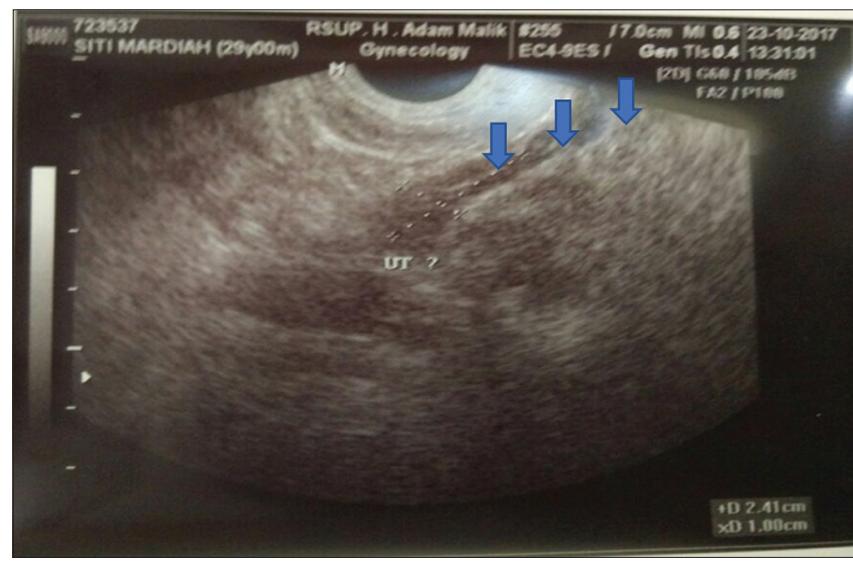

Figure 1: Transvaginal sonography of the internal genitalia uterine aplasia

\section{Discussion}

MRKH syndrome is characterized by the absent or hypoplastic uterus and vagina. Failure of fusion and development of Mullerian ducts result in muscular thickening at the proximal end of each tube that is joined in the midline by a visible and palpable cord resembling hypoplastic bicornuate uterus without an endometrial lining. The typical clinical presentation of this syndrome is primary amenorrhea, in association or not with cyclic colic pain, in an adolescent with secondary sexual characteristics compatible with age, with no sign of virilization. Gynecologic examination may detect either absence of the vaginal canal or vaginal shortening [1], [2].

Most laboratories with low-resource settings do not have the facility to measure FSH, estradiol, testosterone, thyroid-stimulating hormone, and prolactin. This hormonal examination is routinely used in the diagnosis of amenorrhea in a clinical setting with high resources [3], [4].

Imaging studies such as ultrasonography and magnetic resonance imaging, in association or not with laparoscopy, are necessary to allow the determination of the anatomic characteristics of the syndrome. Ultrasonography is the initial method of choice. This method can demonstrate the absence of the uterus between the bladder and the rectum [5]. The vestigial lamina may be confused with the uterus, as it is found in its habitual site. Furthermore, renal anomalies may be observed in cases of type II syndrome [5], [6], [7].

Magnetic resonance imaging is the most sensitive and specific imaging method in the evaluation of this syndrome not only for allowing the acquisition of multiplanar images but also for allowing the acquisition of sequences with fat saturation. It allows a good definition of anatomical alterations such as uterine agenesis, as well as evaluating ovaries, vagina, and associated anomalies [5], [6], [7].

Laparoscopy is indicated only in cases where the evaluation by the two previous imaging methods is inconclusive and provided this method allows the definitive therapy. Once the diagnosis of MRKH syndrome is established, a clinical investigation should be undertaken to identify possible associated malformations [5], [6], [7]. The final diagnosis is achieved by the association of the imaging findings with the presence of the karyotype 46, XX [8].

The differential diagnosis should be made with other situations where the patient presents primary amenorrhea and normal secondary sexual characteristics, such as congenital absence of uterus and vagina, isolate vaginal atresia with androgen insensitivity syndrome, and transverse vaginal septum with imperforate hymen [8], [9].

The indicated anatomic treatment is the surgical or non-surgical creation of a neovaginal, which may allow these patients to have a normal sex life [6]. As the surgical approach is chosen, uterine remnants can be removed to avoid future endometriosis [9].

Patients who want to have children should be encouraged to adopt, or the possibility of having biological children by means of assisted reproduction techniques should be suggested, considering that the presence of functional ovaries in these women allows the production of normal ovules [2], [9].

Surgical treatment of MRKH syndrome is achieved by vaginal reconstruction, which includes; Williams vaginoplasty, which includes sewing the labia majora into a perineal sac, but the vagina made is external, short, and unsatisfactory for penetrative sexual intercourse; this procedure is no longer practiced. The Vecchietti procedure consists of increasing the size of the vagina by gradually applying traction to the vaginal wall. Finally, neovagina can be made in the chambers of the physic and coated by different tissues such as skin (Mclndoe-Reed), peritoneum (Davydov), and intestine [2], [9]. Even with the recent developments in the management of this syndrome, its diagnosis causes 
significant psychological distress, affecting the patients' quality of life because of the absence of menstruation and impossibility of pregnancy, thus requiring a multidisciplinary approach. The distress caused by the diagnostic may be alleviated by surgical or non-surgical treatments, by the passage of time, by counseling, by family's support, and by support groups [9], [10].

\section{Conclusions}

MRKH syndrome is a rare disorder that affects woman. It is characterized by the failure of the uterus and the vagina to develop properly in women who have normal ovarian function and normal external genitalia.

\section{Declarations}

\section{Authors' contributions}

MR contributed in manuscript writing and editor, Dlutan in manuscript writing, MA in data collection, and AYMR in drafting of manuscript. All authors have read and approved the final version of the manuscript.

\section{Acknowledgments}

The authors would like to thank Obstetrics and Gynecology Department of Universitas Sumatera Utara for the support.

\section{Consent for publication}

Written informed consent for publication of their clinical details and/or clinical images was obtained from the patient. A copy of the consent form is available for review by the editor of this journal.

\section{Availability of data and materials}

The data and materials will not be shared because of patient privacy.

\section{References}

1. Morcel K, Camborieux L. Programme de recherches sur les aplasies müllériennes, Guerrier, D. Mayer-Rokitansky-KusterHauser (MRKH) syndrome. Orphanet J Rare Dis. 2007;14:2-13. https://doi.org/10.1186/1750-1172-2-13

2. Jabeen M. Mayer-Rokitansky-Kuster-Hauser syndrome. World J Laparosc Surg. 2011;4(2):123-8.

3. Lagro M. Gynecology for Less-Resourced Locations: Amenorrhea. London: Sapiens Publishing; 2012. p. 84-90.

4. Gordon CM. Clinical practice: Functional hypothalamic amenorrhea. N Engl J Med. 2010;363(4):365-71.

PMid:20660404

5. The Practice Committee of the American Society of Reproductive Medicine: Current Evaluation of Amenorrhea. Fertil Steril Available from: https://www.asrm.org/topics/topicsindex/infertility. [Last accessed on 2019 May 05].

6. Nelson LM. Clinical practice: Primary ovarian insufficiency. $\mathrm{N}$ Engl J Med. 2009;360(6):606-14.

PMid: 19196677

7. Klein DA, Poth MA. Amenorrhea: An approach to diagnosis and management. Am Fam Physician. 2013;87(11):781-8. PMid:23939500

8. Master-Hunter T, Heiman DL. Amenorrhea: Evaluation and treatment. Am Fam Physician. 2006;73(8):1374-82. PMid:16669559

9. Deligeoroglou E, Athanasopoulos N, Tsimaris P, Dimopoulos KD, Vrachnis N, Creatsas G. Evaluation and management of adolescent amenorrhea. Ann N Y Acad Sci. 2010;1205:23-32. https://doi.org/10.1111/j.1749-6632.2010.05669.x PMid:20840249

10. Rusda M. Do We Need To Treat Any of Uterine Abnormality in Fertility Seeking Patient? The Universitas Sumatera Utara Institutional Repository; 2015. 